\title{
Comunicação científica sob o espectro da Ciência Aberta: um modelo conceitual contemporâneo
}

\author{
Scientific communication under the Open Science spectrum: a \\ contemporary conceptual model
}

\section{Comunicación científica bajo el espectro de la ciência aberta: um modelo conceptual contemporâneo}

\author{
Danyelle Mayara Silva | danielemaiara@gmail.com \\ Universidade de Brasília, Faculdade de Ciência da Informação, Programa de Pós-Graduação em Ciência da \\ Informação. Brasília, DF, Brasil.
}

\author{
Elton Mártires Pinto | eltomrs@gmail.com \\ Universidade de Brasília, Faculdade de Ciência da Informação, Programa de Pós-Graduação em Ciência da \\ Informação. Brasília, DF, Brasil.

\section{Érika Rayanne Silva de Carvalho | erikaunb@gmail.com \\ Universidade de Brasília, Faculdade de Ciência da Informação, Programa de Pós-Graduação em Ciência da Informação. Brasília, DF, Brasil.}

\section{Patrícia Resende Pereira | patriciaresendeeduc@hotmail.com \\ Universidade de Brasília, Faculdade de Ciência da Informação, Programa de Pós-Graduação em Ciência da Informação. Brasília, DF, Brasil.}

Fernando César Lima Leite | fernandodfc@gmail.com

Universidade de Brasília, Faculdade de Ciência da Informação, Programa de Pós-Graduação em Ciência da Informação. Brasília, DF, Brasil.

\section{Resumo}

A disseminação da informação científica tem ganhado celeridade e eficácia, sobretudo, pelo desenvolvimento das modernas tecnologias da informação e comunicação. O uso massivo dessas tecnologias para coleta, tratamento e análise de dados vem criando o que alguns autores, entre eles Tim Gray, chamam de quarto paradigma da ciência, ou eScience. Atualmente, a adoção de práticas ligadas à Ciência Aberta tem provocado forte impacto na forma como os pesquisadores comunicam o desenvolvimento de suas pesquisas. Nesse contexto, este trabalho propõe um modelo conceitual de comunicação científica sob o espectro dos conceitos relativos à Ciência Aberta. O trabalho demonstra que as práticas de comunicação científica descritas por modelos convencionais, como o de Garvey e Griffith e o idealizado por Hurd, servem de base para a análise da comunicação científica contemporânea, porém necessitam ser adaptados à realidade atual.

Palavras-chave: Comunicação científica; Informação científica; Ciência aberta; Modelo conceitual. 


\section{Abstract}

The dissemination of scientific information has become faster and more efficient, above all, through the development of modern information and communication technologies. The widespread use of these technologies for data collection, processing and analysis has created what authors like Tim Gray call the fourth paradigm of science, or eScience. Today, practices related to Open Science have had a powerful impact on how researchers communicate their findings. On this basis, this paper proposes a conceptual model of scientific communication inspired by the concepts of Open Science. It shows that the practices of scientific communication described by conventional models, such as those of Garvey and Griffith and idealized by Hurd, can serve as a basis for analyzing contemporary scientific communication, but they must be adapted to the current reality

Keywords: Scientific communication; Scientific information; Open Science; Conceptual model.

\section{Resumen}

La diseminación de la información científica ha ganado celeridad y eficacia, sobre todo, por el desarrollo de las modernas tecnologías de la información y comunicación. El uso masivo de estas tecnologías para la recolección, tratamiento y análisis de datos viene creando lo que algunos autores, entre ellos Tim Gray, llaman el cuarto paradigma de la ciencia, o eScience. Actualmente, la adopción de prácticas vinculadas a la Ciencia Abierta ha provocado un fuerte impacto en la forma en que los investigadores comunican el desarrollo de sus trabajos científicos. En este contexto, este artículo propone un modelo conceptual de comunicación científica bajo el espectro de los conceptos relativos a la Ciencia Abierta. El trabajo demuestra que las prácticas de comunicación científica descritas por modelos convencionales, como el de Garvey y Griffith y el idealizado por Hurd, sirven de base para el análisis de la comunicación científica contemporánea, no obstante, necesitan adaptarse a la realidad actual.

Palabras-clave: Comunicación científica; Información científica; Ciencia aberta; Modelo conceptual.

\section{Proposta}

\section{Contexto}

São intrínsecas à ciência a atividade de recorrer ao conhecimento previamente obtido sobre o fenômeno eleito para a investigação e a exposição das descobertas dela decorrentes. Dados gerados e/ou obtidos, anotações de pesquisa, registros de projetos, descrições de procedimentos metodológicos e outros recursos também compõem o grupo de artefatos informacionais que trazem consigo potencial para alimentar novos ciclos de produção do conhecimento e, portanto, são de interesse da comunidade científica. Toda essa miríade de práticas informacionais ocorre por meio de processos de comunicação. É por essa razão que Meadows ${ }^{1}$ afirma que a comunicação encontra-se no coração da ciência.

A comunicação científica obteve grandes avanços decorrentes do desenvolvimento acelerado e adoção de tecnologias de informação e comunicação (TIC) a partir da segunda metade do século XX. Esse período tem como características as profundas alterações sociais, a emergência do fenômeno da Big Science e a produção de novas tecnologias, de tal modo que disciplinas científicas, especialidades e áreas do conhecimento têm evoluído em razão de contribuições dos novos meios, da integração de recursos, da expansão das redes de telecomunicação e do uso computacional no gerenciamento da informação para o alargamento da cognição humana².

A criação da Internet, em especial, teve um forte impacto sobre a forma como pesquisadores produzem, disseminam e usam os resultados da investigação científica. Por meio do desenvolvimento da Web é que há quinze anos tornou-se possível à comunidade científica estabelecer novos padrões de comunicação de modo que o acesso sobrepujasse as barreiras financeiras, técnicas e legais que até então eram impostas aos 
pesquisadores 3 . O movimento mundial em prol ao acesso aberto à informação científica tem desempenhado um importante papel na proposição de novas formas de comunicar os resultados de pesquisa e isso pode ser observado por meio de resultados de diversos estudos, como os dos autores Tennant, ${ }^{4}$ Xianwen, ${ }^{5} \mathrm{e}$ Hitchcock, ${ }^{6}$ além da identificação do crescimento de revistas científicas de acesso aberto e repositórios digitais que disponibilizam textos completos livres de custos.

A preocupação com a diversidade de artefatos informacionais necessários e produzidos nas atividades de pesquisa não é nova. Elaborado em 1971, o modelo denominado UNISIST7, além dos canais formais e informais de comunicação previu os canais tabulares. A partir desses meios, segundo o modelo, dados gerados e/ou obtidos ao longo das pesquisas seriam gerenciados e distribuídos por meio de unidades organizacionais, denominadas de centros de dados. Além dessa, outras iniciativas isoladas foram conduzidas ao longo do século XX, com sucesso em alguns projetos de disciplinas específicas.

No entanto, mais recentemente a comunidade científica e até mesmo o movimento de acesso aberto têm se confrontado com uma nova necessidade de reflexão e adequação quanto às formas de promoção da comunicação científica, a fim de alcançar maior efetividade com os seus esforços. Isso porque as prática atuais se baseiam no uso intensivo de dados e as publicações que descrevem e apresentam os resultados de pesquisas constituem apenas uma parte da cadeia de produção do conhecimento científico, chegando até ser frequentemente comparada à ponta de um iceberg diante da magnitude de todas as outras etapas. Segundo Jim Gray (2007, apud TOLLE, TANSLEY, HEY, 2011, p. 24) ${ }^{8}$, não basta garantir o acesso a este tipo de publicação em uma época em que "a internet pode fazer mais do que simplesmente disponibilizar o texto completo de artigos de pesquisa. Em princípio, ela pode unificar todos os dados científicos e toda a literatura para criar um mundo em que os dados e a literatura possam interagir".

Desta forma, o sistema de comunicação científica deve contemplar não apenas a publicação dos resultados alcançados, a fim de permitir que os pesquisadores possam ser capazes de compartilhar procedimentos e avanços no processo científico pari passu a sua consecução. Sob os aspectos do quarto paradigma da ciência, a chamada eScience, a execução das atividades científicas passa a ser mais colaborativa, transparente e célere. No escopo do que passa a ser compartilhado entre os cientistas estão os dados brutos de pesquisa (open data), anotações (open notebooks), laboratórios, instrumentos e ferramentas de pesquisa (collaboratories e research tools), registros de projetos de pesquisa entre outros.

A partilha desses itens é possível por meio das infraestruturas digitais baseadas nos protocolos de comunicação na Internet. Assim, tanto aqueles modelos que representam processos de comunicação científica fundamentalmente baseados no meio analógico/impresso quanto aqueles modelos que consideram preponderantemente ou exclusivamente o fluxo da informação científica sob a ótica da publicação, precisam ser revistos. Isso porque devem, ao mesmo tempo, levar em consideração a realidade de execução de atividades de uso, produção e disseminação da informação científica em ambientes completamente digitais e a diversidade de artefatos informacionais produzidos e necessários para o alargamento de novos ciclos de produção do conhecimento científico. Exemplo de modelo de comunicação científica que foi capaz de mapear desde o início do processo de pesquisa até a divulgação dos seus resultados foi o proposto por William Garvey e Belver Griffith ${ }^{9}$ na década de 1970. Este modelo, que foi utilizado por muitos pesquisadores e aplicado a diferentes áreas do conhecimento, não contempla as possibilidades que seriam criadas pelas tecnologias produzidas nos anos vindouros. Hurd $^{10}$ (1996) já havia alertado que desde a proposição do modelo de Garvey e Griffith a emergência das tecnologias da informação haviam alterado e enriquecido, de maneira drástica, as opções de processos de comunicação. A autora, por sua vez propôs um modelo de representação de processos de comunicação científica estimando como estes seriam no ano de $2020^{11}$. Sua proposta considera as modernas tecnologias e a disponibilização e preservação de mais do que apenas os relatórios intermediários e finais da pesquisa. Ou seja, seu modelo já contempla os dados de pesquisa e o uso de recursos compartilhados, como é o caso dos colaboratórios. 
No entanto, a emergência das práticas da eScience e a adoção cada vez mais intensa por parte da comunidade científica dos princípios que norteiam a execução das atividades de pesquisa sob o espectro da Ciência Aberta torna necessária a compreensão das dinâmicas e fluxos que interferem na comunicação científica na contemporaneidade. Analisar o papel dos atores que se relacionam a este processo, seus comportamentos e processos à luz das práticas atuais e das possibilidades trazidas pelas TIC tem potencial para criar um corpo de conhecimento orientador para o desenvolvimento de políticas de fomento à ampliação de disponibilidade, acesso e uso mais eficazes dos conteúdos científicos, além da promoção de produtos e serviços mais alinhados às necessidades da comunidade científica.

\section{Problema}

Considerando as novas práticas de condução da pesquisa científica e as necessidades dela decorrentes, este trabalho propõe e apresenta, por meio de modelo conceitual, a comunicação científica sob o espectro das atividades de Ciência Aberta.

\section{Abordagem}

Segundo Case e Given ${ }^{12}$, modelos são geralmente usados para focar questões específicas. Nesse contexto, propõe-se um modelo que representa a comunicação científica sob o conjunto dos conceitos relativos à Ciência Aberta, considerando que essas novas práticas afetam profundamente os processos de comunicação entre os pares científicos.

Fig. 1: Modelo de comunicação científica sob o espectro da Ciência Aberta

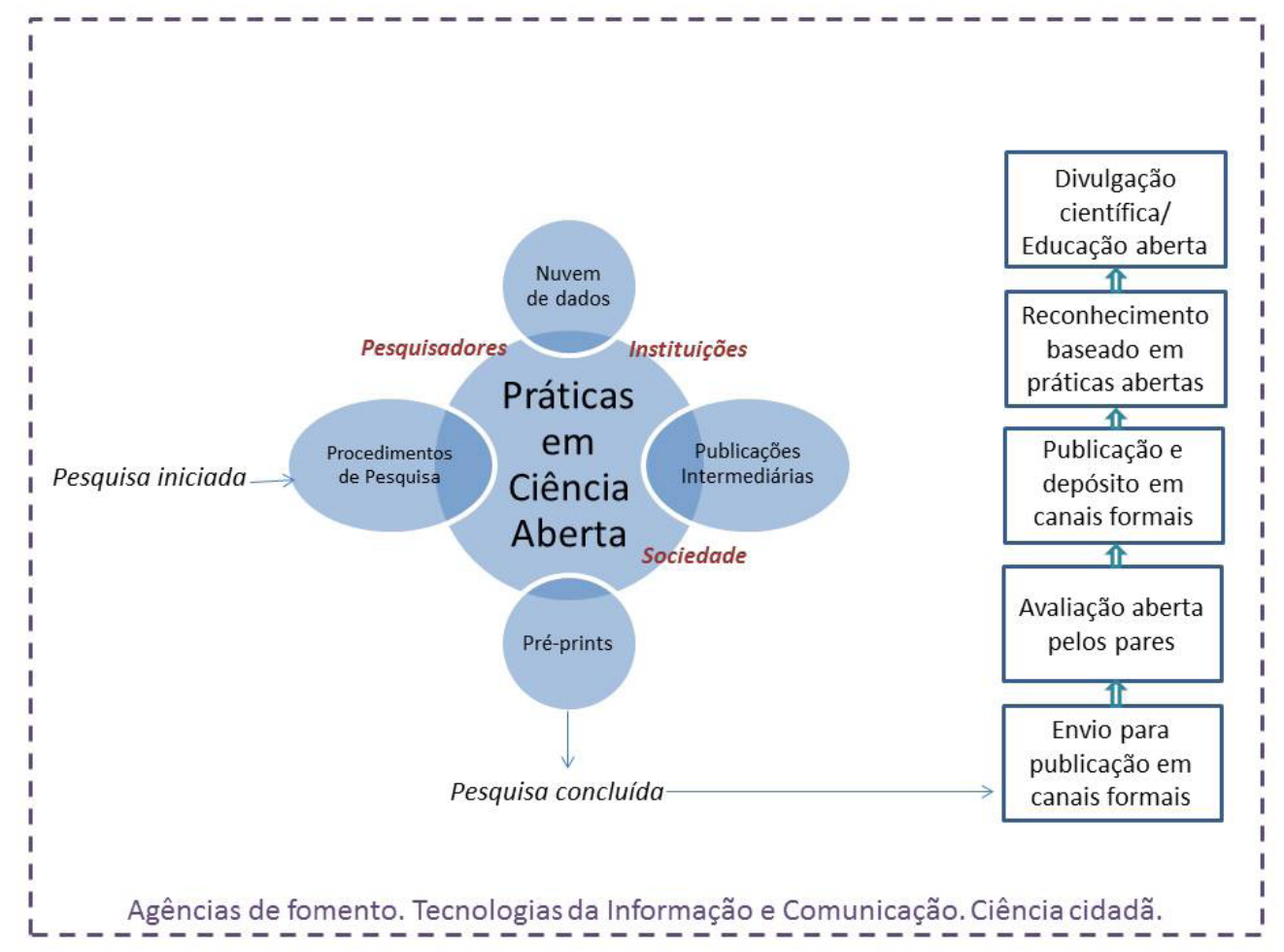

Fonte: elaboração própria. 
Resumidamente, considerando os conceitos embutidos e relacionados no modelo conceitual, logo após o início da pesquisa já é possível observar algumas práticas colaborativas de Ciência Aberta, destacando-se: procedimentos de pesquisa adotados, que se relacionam ao projeto de pesquisa a ser desenvolvido; nuvens de dados criadas a partir das coletas e desenvolvimento das investigações; produção das publicações intermediárias; e pré-prints, que devem ser depositados em repositórios digitais de acesso aberto.

Estas práticas em Ciência Aberta envolvem diversos atores, tais como: os pesquisadores, as instituições e a sociedade. Sob os princípios da Ciência Aberta é importante destacar o papel da sociedade enquanto ente interessado no desenvolvimento de pesquisas e no necessário acesso aos seus frutos como parte do retorno do investimento de recursos nas mesmas. As agências de fomento, por sua vez, exercem papel fundamental no estímulo à pesquisa uma vez que possuem o papel de financiadores e sustentadores da atividade científica. Por essa razão, como ilustrado no modelo, acredita-se que sua influência esteja presente em todas as etapas do processo. Após o desenvolvimento, a pesquisa é concluída e, por conseguinte, gera resultados. Logo, reitera-se as recomendações da Royal Society apresentadas no relatório "Science as an open enterprise" de que os cientistas devem comunicar os dados coletados e os modelos criados, permitindo assim, acesso livre, aberto, de forma inteligível, avaliável e utilizável a outros especialistas da mesma área/campo de qualquer lugar do mundo.

As próximas etapas presentes no modelo otimizam o tempo e alteram os canais tradicionais de veiculação das pesquisas. Inicialmente, o pesquisador envia o manuscrito para publicação em canais formais, como por exemplo, os periódicos científicos. Nesses canais ocorre a atividade de validação do conhecimento científico, sob responsabilidade de outros pesquisadores, entendida aqui, como avaliação aberta. Em seguida, o manuscrito é publicado e depositado nos canais de comunicação por meio de duas vias: dourada e verde. Em seguida, observa-se o reconhecimento baseado em práticas abertas, que se dá, sobretudo, por meio dos comentários da comunidade científica e de índices de impacto relacionados à publicação. Esse reconhecimento amplia as avaliações pelos pares, haja vista que outros pesquisadores, simultaneamente, fazem suas avaliações, contribuindo assim para a melhoria da publicação.

As pesquisas elaboradas em práticas de Ciência Aberta agregam conhecimentos no contexto da ciência e geram insumos para novas pesquisas. Todavia, também cumprem um papel social, à medida que a divulgação dos resultados da pesquisa possibilita que as informações científicas produzidas se tornem mais acessíveis e compreensíveis à sociedade como um todo. Iniciativas no âmbito da Educação Aberta cooperam para a divulgação científica, uma vez que promovem a aprendizagem por diversos mecanismos, com vistas à inclusão social.

\section{Principais conclusões}

É possível apontar que a adoção das práticas alinhadas à Ciência Aberta e à eScience tem transformado a maneira como os pesquisadores produzem ciência. Como consequência, a forma de comunicar os conteúdos científicos aos pares também tem sofrido fortes alterações. Estar atento a essas mudanças e considerar o efeito das tecnologias da informação e da comunicação, o papel da sociedade no contexto de desenvolvimento da pesquisa e o comportamento dos pesquisadores quanto ao compartilhamento de recursos é um imperativo para compreender os processos de comunicação científica na atualidade.

Conforme Meadows ${ }^{14}$ (1999, p. viii), "as mudanças que afetam o mundo científico como um todo não causam necessariamente impactos idênticos na comunicação de pesquisas em ciências e humanidades” e por essa razão a representação gráfica de um modelo conceitual pode conter disparidades com áreas do conhecimento específicas. No entanto, com esse trabalho buscou-se propor um modelo que reflita aspectos gerais da comunicação científica sob o espectro da Ciência Aberta no século XXI. 


\section{Referências}

1. Meadows AJ. Communicating research. San Diego: Academic Press; 1998.

2. Crawford SY. Scientific communication and the growth of Big Science. In: Crawford SY, Hurd JM, Willer AC. From print to electronic: the transformation of scientific communication. Medford (NJ): Information Today; 1996. p. 1-8.

3. Chan L, Cuplinskas D, Eisen M, Friend F, Genova Y, Guédon JC, et al. Budapest open access initiative declaration [Internet]. 2002. [citado em 20 abr. 2017]. Disponível em: http://www. budapestopenaccessinitiative.org/read.

4. Tennant JP, Waldner F, Jacques DC, Masuzzo P, Collister, LB, Hartgerink CHJ. The academic, economic and societal impacts of Open Access: an evidence-based review. F1000Research [Internet]. 2016 [citado em 21 abr. 2017]; 5:1-55. Disponível em: https://f1000research.com/articles/5-632/v3.

5. Xianwen W, Chen L, Wenli M, Zhichao F. The open access advantage considering citation, article usage and social media attention. Scientometrics [Internet]. 2015 [citado em 21 abr. 2017]; 103(2):555-64. Disponível em: link.springer.com/article/10.1007\%2Fs11192-015-1547-0.

6. Hitchcock S. The effect of open access and downloads ('hits') on citation impact: a bibliography of studies [Internet]. 2013. [citado em 21 abr. 2017]. Disponível em: https://eprints.soton.ac.uk/354006/1/ oacitation-biblio-snapshot0613.html\#indexes.

7. Organização das Nações Unidas para a Educação, Ciência e da Cultura. Unisist: study report on the feasibility of a world science information system. Paris: Unesco; 1971.

8. Hey T, Tansley S, Tolle K. O Quarto Paradigma: descobertas científicas na era da eScience. São Paulo: Oficina de Textos; 2011. Jim Gray e a eScience: um método científico transformado.

9. Garvey WD, Griffith BC. Scientific communication as a social system. In: Communication: the essence of science. London: Pergamon Press; 1979. p. 148-64.

10. Hurd JM. Models of scientific communications systems. In: Crawford SY, Hurd JM, Willer AC. From print to electronic: the transformation of scientific communication. Medford, (NJ): Information Today; 1996. p. 9-33.

11. Hurd JM. The transformation of scientific communication: a model for 2020. Journal of the American society for information science [Internet]. 2000 [citado em 21 abr. 2017]; 51(14):1279-83. Disponível em: https://pdfs.semanticscholar.org/51c0/3c5ce7757ac89f22cb7559f85caded54c75c.pdf.

12. Case DO, Given LM. Looking for information: a survey of research on information seeking, needs, and behaviour. UK: Emerald; 2016.

13. Boulton G, Collins B, Elias P, Hall DW, Laurie G, O’Neill O. Science as an open enterprise [Internet]. Londres: The Royal Society; 2012 [citado em 20 abr. 2017]. Disponível em: https://royalsociety.org/ / media/policy/projects/sape/2012-06-20-saoe.pdf.

14. Meadows AJ. A comunicação científica. Brasília: Briquet de Lemos; 1999. 